Noname manuscript No.

(will be inserted by the editor)

\title{
Bernstein dual-Petrov-Galerkin method: application to 2D time fractional diffusion equation
}

\author{
M. Jani · S. Javadi • E. Babolian • D. Bhatta
}

the date of receipt and acceptance should be inserted later

\begin{abstract}
In this paper, we develop a Bernstein dual-Petrov-Galerkin method for the numerical simulation of a two-dimensional fractional diffusion equation. A spectral discretization is applied by introducing suitable combinations of dual Bernstein polynomials as the test functions and the Bernstein polynomials as the trial ones. We derive the exact sparse operational matrix of differentiation for the dual Bernstein basis which provides a matrix based approach for the spatial discretization. It is shown that the method leads to banded linear systems that can be solved efficiently. The stability and convergence of the proposed method is discussed. Finally, some numerical examples are provided to support the theoretical claims and to show the accuracy and efficiency of the method.
\end{abstract}

Keywords Fractional PDEs · Bernstein polynomials · 2D Subdiffusion · dual-Petrov-Galerkin · Dual Bernstein basis . Operational matrix

Mathematics Subject Classification (2010) 41A10 $\cdot 65 \mathrm{M} 22 \cdot 35 \mathrm{R} 11 \cdot 76 \mathrm{M} 22$

\section{Introduction}

Bernstein polynomial basis plays an important role in computer graphics for geometric modeling, curve and surface approximation. Some interesting features have been investigated for this basis in the last decades; for instance, it is proven to be an optimal stable basis among nonnegative bases in a sense described in [8]. Also, it provides some optimal shape preserving features [2]. We refer to [5, 6, 7] for detailed properties and applications in computer aided geometric design (CAGD).

Bernstein basis has also been used for the numerical solution of differential, integral, integrodifferential and fractional differential equations, see e.g. [1, 13, 14, 21, 28] and the references therein.

M. Jani · S. Javadi · E. Babolian

Department of Mathematics, Faculty of Mathematical Sciences and Computer, Kharazmi University, Tehran, Iran E-mail: mostafa.jani@gmail.com javadi@khu.ac.ir babolian@khu.ac.ir.

D. Bhatta

School of Mathematical \& Statistical Sciences, The University of Texas Rio Grande Valley, 1201 West University Drive, Edinburg, TX, USA

E-mail: dambaru.bhatta@utrgv.edu 
However, it is not orthogonal and so leads to dense linear systems when using in numerical methods. Some numerical approaches implement the orthogonalized Bernstein basis. However, as we will see in the next section, it fails to keep some interesting properties of the Bernstein basis. Another approach uses the dual Bernstein polynomials (DBP) introduced by Juttler in 1998 [18. To the best of our knowledge, the DBP basis has been only discussed from the CAGD point of view (see the works of Lewanowicz and Wozny e.g. [19, 33]). So it is of interest to explore some new aspects of this basis in order to facilitate the numerical methods for differential equations that are based on Bernstein polynomials and to present a method for time fractional diffusion equation in two dimensions.

Fractional partial differential equations (FPDEs) have been widely used for the description of some important physical phenomena in many applied fields including viscoelastic materials, control systems, polymer, electrical circuits, continuum and statistical mechanics, etc., see, for instance [3, 11, 22, 23, 34] and the references therein. The subdiffusion equation is a FPDE describing the behavior of anomalous diffusive systems with the probability density of particles diffusing proportional to the mean square displacement $\chi^{2}(t) \propto t^{\alpha}$ with $0<\alpha<1$ [9. Anomalous diffusion equations have been used for modeling transport dynamics, especially the continuous time random walk, the contamination in porous media, viscoelastic diffusion, etc 9, 10, 11, 22, 32. For the numerical solution of the one-dimensional problem, we refer to [15, 27, 31, 41] and the references therein. Some classic numerical methods for PDEs have been developed for the simulation of two-dimensional subdiffusion equation, for example the finite difference schemes [10, 25, 27, meshless methods 30, 35, finite element method [40, alternating direction implicit methods [38, 39, etc.

In this paper, deriving some new aspects of DBPs, we present suitable combinations of these functions in order to develop a dual-Petrov-Galerkin method for solving the following 2D subdiffusion equation [32, 35, 36, 38, 39, 40.

$$
D_{t}^{\alpha} u(x, y, t)=\kappa \Delta u(x, y, t)+S(x, y, t), \quad(x, y, t) \in \Omega \times(0, T],
$$

with the following initial and boundary conditions

$$
\begin{aligned}
& u(x, y, 0)=g(x, y), \quad(x, y) \in \Omega \\
& u(x, y, t)=0, \quad(x, y, t) \in \partial \Omega \times(0, T]
\end{aligned}
$$

where $\Omega=(0,1)^{2} \subset \mathbb{R}^{2}, \Delta$ is the Laplacian operator, $T>0, \kappa$ is the diffusion coefficient and $S$ is the source function. Here, $D_{t}^{\alpha} u$ denotes the Caputo fractional derivative of order $\alpha, 0<\alpha<1$, with respect to $t$ defined as

$$
D_{t}^{\alpha} u(x, t)=\frac{1}{\Gamma(1-\alpha)} \int_{0}^{t} \frac{1}{(t-s)^{\alpha}} \frac{\partial u(x, s)}{\partial s} d s, \quad 0<\alpha<1 .
$$

The main contribution of our work is the development of an accurate Bernstein dual-PetrovGalerkin method and the application for the numerical simulation of the $2 \mathrm{D}$ subdiffusion equation. It is shown the method leads to sparse linear systems. To give a matrix approach of the method, we present some results concerning the DBPs including a recurrence formula for the derivative, constructing a new basis using DBPs, deriving the operational matrix of differentiation and also providing the transformation matrices between the DBPs and the new basis.

The paper is organized as follows: Section 2 presents some new aspects of DBPs and provides modal basis functions and the associated transformation matrices between the bases. Section 3 is devoted to the Bernstein-spectral formulation of the subdiffusion problem [1.1)-(1.3) and the stability and convergence results are discussed in Section 4 Numerical examples are provided in Section 5 . The paper ends with some concluding remarks in Section 6 


\section{Bernstein polynomials and DBPs}

The Bernstein polynomials with degree $N$ on the unit interval are defined by

$$
\phi_{i}(x)=\left(\begin{array}{c}
N \\
i
\end{array}\right) x^{i}(1-x)^{N-i}, \quad 0 \leq i \leq N .
$$

The set $\left\{\phi_{i}(x): i=0, \ldots, N\right\}$ forms a basis for $\mathbb{P}_{N}$, the space of polynomials of degree not exceeding $N$.

These polynomials possess end-point interpolation property, i.e.,

$$
\phi_{i}(0)=\delta_{i, 0}, \quad \phi_{i}(1)=\delta_{i, N}, \quad 0 \leq i \leq N, N>0 .
$$

Also, the summation is one and the integral over the unit interval is constant, namely

$$
\sum_{i=0}^{N} \phi_{i}(x) \equiv 1, \quad \int_{0}^{1} \phi_{i}(x)=\frac{1}{N+1}, i=0,1, \ldots, N .
$$

The derivative enjoys the three-term recurrence relation [12]

$$
\phi_{i}^{\prime}(x)=(N-i+1) \phi_{i-1}(x)-(N-2 i) \phi_{i}(x)-(i+1) \phi_{i+1}(x), \quad 0 \leq i \leq N,
$$

where we adopt the convention that $\phi_{i}(x) \equiv 0$ for $i<0$ and $i>N$.

As we mentioned in the preceding section, the Bernstein basis is not orthogonal. The corresponding orthogonalized basis, obtained e.g., by the Gram-Schmidt process fails to keep some interesting aspects of the original basis. We will not consider this basis in the present work. Instead we turn to the dual basis.

The DBPs are defined as

$$
\tilde{\psi}_{i}(x)=\sum_{j=0}^{N} c_{i, j} \phi_{j}(x)
$$

with the coefficients given by

$$
c_{i, j}=\frac{(-1)^{i+j}}{\left(\begin{array}{c}
N \\
i
\end{array}\right)\left(\begin{array}{c}
N \\
j
\end{array}\right)} \sum_{r=0}^{\min (i, j)}(2 r+1)\left(\begin{array}{c}
N+r+1 \\
N-i
\end{array}\right)\left(\begin{array}{c}
N-r \\
N-i
\end{array}\right)\left(\begin{array}{c}
N+r+1 \\
N-j
\end{array}\right)\left(\begin{array}{c}
N-r \\
N-j
\end{array}\right) .
$$

It is verified that they satisfying the biorthogonal system [18, Theorem 3]

$$
\int_{0}^{1} \phi_{i}(x) \tilde{\psi}_{j}(x) d x=\delta_{i j}, \quad 0 \leq i, j \leq N
$$

It is worth noting that less than a quarter of the entries of transformation matrix between the Bernstein and dual Bernstein basis $C=\left[c_{i, j}\right]$, are to be computed directly by $[2.5)$; for it is bisymmetric, i.e., symmetric about both of the main diagonal and antidiagonal.

Another property which is used later is that the sum of the entries for each row (column) is equal to the order of the matrix, i.e.,

$$
\sum_{i=0}^{N} c_{i, j}=\sum_{j=0}^{N} c_{i, j}=N+1 .
$$

In the next lemma, we present some properties of the DBPs. 
Lemma 1 Let $N$ be a nonnegative integer. The following statements hold.

(i) For all $x \in[0,1], \tilde{\psi}_{N-i}(x)=\tilde{\psi}_{i}(1-x), 0 \leq i \leq N$.

(ii) For all $x \in[0,1], \sum_{i=0}^{N} \tilde{\psi}_{i}(x)=N+1$.

(iii) The basis functions have the same definite integral, i.e., $\int_{0}^{1} \tilde{\psi}_{i}(x) d x=1, \quad 0 \leq i \leq N$.

Proof The first statement is an immediate consequence of the similar formula for Bernstein polynomials, i.e., $\phi_{N-i}(x)=\phi_{i}(1-x)$. From 2.4 , 2.7) and 2.2, we have

$$
\begin{aligned}
\sum_{i=0}^{N} \tilde{\psi}_{i}(x) & =\sum_{i=0}^{N} \sum_{j=0}^{N} c_{i, j} \phi_{j}(x) \\
& =\sum_{j=0}^{N} \phi_{j}(x) \sum_{i=0}^{N} c_{i, j}=N+1 .
\end{aligned}
$$

statement (iii) is also verified similarly.

The property (i) implies that $\tilde{\psi}_{i}$, for $\left[\frac{N}{2}\right]+1 \leq i \leq N$, need not to be computed directly by 2.4-2.5. It especially gives $\tilde{\psi}_{i}(0)=\tilde{\psi}_{N-i}(1)$.

\subsection{Modal basis functions}

One may choose a suitable compact combinations of orthogonal polynomials as the trial and test basis for the Galerkin and Petrov-Galerkin methods for BVPs in such a way leading to sparse linear systems for some special problems (see e.g., [17, 37]). Here, we use this idea for the non-orthogonal Bernstein polynomials to present a simple and accurate dual-Petrov-Galerkin spectral method for two-dimensional subdiffusion equation. Following Shen, et. al. [17 and 29, Section 1.3], we will refer to such basis functions as the modal basis functions.

Proposition 1 Let $N \geq 2$ be an integer, $\left\{\tilde{\psi}_{i}: 0 \leq i \leq N\right\}$ be the dual Bernstein basis and $\mathbb{P}_{N}^{0}=\{u \in$ $\left.\mathbb{P}_{N}: u(0)=0, u(1)=0\right\}$. Set

$$
\psi_{i}(x)=\tilde{\psi}_{i}(x)+a_{i} \tilde{\psi}_{i+1}(x)+b_{i} \tilde{\psi}_{i+2}(x),
$$

for $0 \leq i \leq N-2$, where

$$
\begin{aligned}
a_{i} & =\frac{2 i+4}{N-i+1} \\
b_{i} & =\frac{(i+2)(i+3)}{(N-i)(N-i+1)}
\end{aligned}
$$

Then, the polynomials $\tilde{\psi}_{i}(x)$ vanish at 0 and 1 , so the set $\left\{\psi_{i}(x)\right\}_{i=0}^{N-2}$ forms a basis for $\mathbb{P}_{N}^{0}$.

Proof By 2.4 and (2.1), we have

$$
\tilde{\psi}_{i}(0)=\sum_{j=0}^{N} c_{i, j} \phi_{j}(0)=c_{i, 0}=(-1)^{i}(N+1)\left(\begin{array}{c}
N+1 \\
i+1
\end{array}\right) .
$$



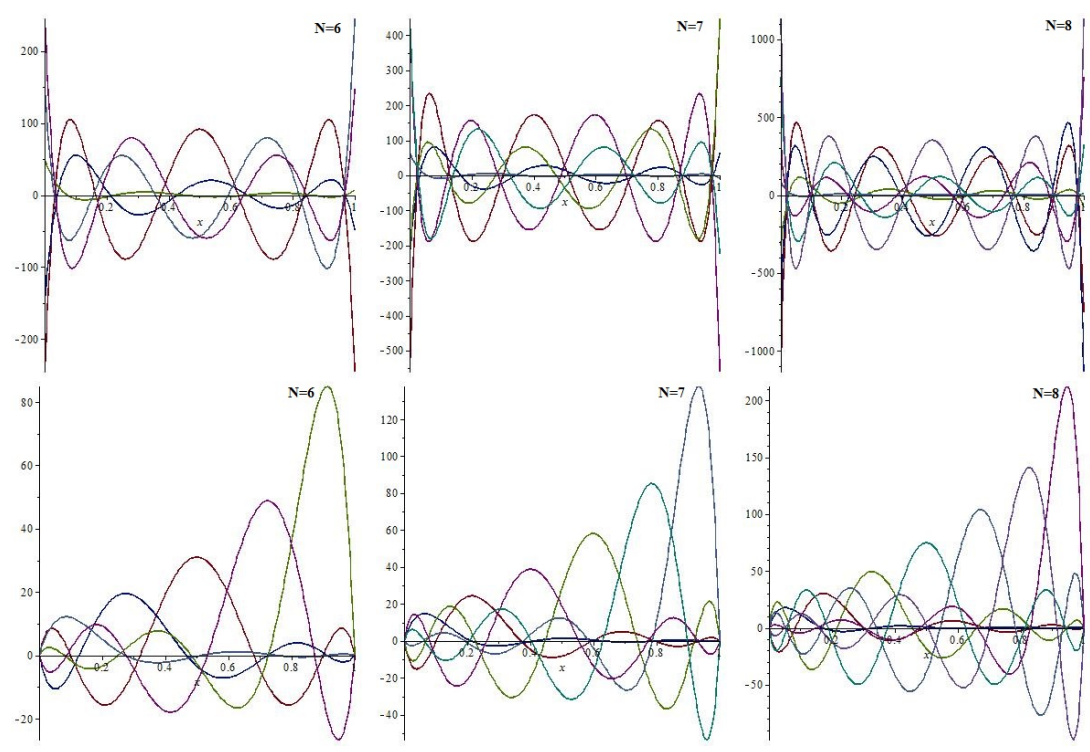

Fig. 2.1 Graphs of DBPs $\left\{\tilde{\psi}_{i}(x), 0 \leq i \leq N\right\}$ (top) and the modal basis functions $\left\{\psi_{i}(x), 0 \leq i \leq N-2\right\}$ (bottom).

From Lemma 1, we infer

$$
\tilde{\psi}_{i}(1)=\tilde{\psi}_{N-i}(0)=(-1)^{N-i}(N+1)\left(\begin{array}{c}
N+1 \\
i
\end{array}\right) .
$$

By 2.10 and 2.11 , we obtain

$$
\begin{aligned}
& \psi_{i}(0)=(-1)^{i}(N+1)\left(\left(\begin{array}{c}
N+1 \\
i+1
\end{array}\right)-a_{i}\left(\begin{array}{c}
N+1 \\
i+2
\end{array}\right)+b_{i}\left(\begin{array}{c}
N+1 \\
i+3
\end{array}\right)\right)=0 \\
& \psi_{i}(1)=(-1)^{N-i}(N+1)\left(\left(\begin{array}{c}
N+1 \\
N-i+1
\end{array}\right)-a_{i}\left(\begin{array}{c}
N+1 \\
N-i
\end{array}\right)+b_{i}\left(\begin{array}{c}
N+1 \\
N-i-1
\end{array}\right)\right)=0
\end{aligned}
$$

for $0 \leq i \leq N-2$. It is easy to see $\left\{\psi_{i}(x)\right\}_{i=0}^{N-2}$ is linearly independent. Since $\operatorname{dim} \mathbb{P}_{N}^{0}=N-1$, this set is a basis for $\mathbb{P}_{N}^{0}$. This completes the proof.

Figure 2.1. illustrates the DBPs and the modal basis functions for $6 \leq N \leq 8$. It is seen that the modal basis functions have less values than the corresponding dual functions on the unit interval, expecting less round-off errors.

\subsection{Transformation matrices and the operational matrix for derivatives}

For $N \geq 2$, consider the $(N+1)$-vector $\tilde{\boldsymbol{\Psi}}$ and the $(N-1)$-vector $\boldsymbol{\Psi}$ consisting of dual functions given by (2.4) and the modal basis functions given by $(2.8)$, respectively:

$$
\begin{aligned}
\tilde{\boldsymbol{\Psi}}(\cdot) & =\left[\tilde{\psi}_{i}(\cdot): 0 \leq i \leq N\right]^{T}, \\
\boldsymbol{\Psi}(\cdot) & =\left[\psi_{i}(\cdot): 0 \leq i \leq N-2\right]^{T} .
\end{aligned}
$$


For simplicity, we ignore the dependence of the vectors on variable. First, note that

$$
\boldsymbol{\Psi}=\mathbf{G} \tilde{\boldsymbol{\Psi}}
$$

where $\mathbf{G}=\left[g_{i, j}\right]$ is an $(N-1) \times(N+1)$ matrix with three diagonals as

$$
g_{i, j}=\left\{\begin{array}{ll}
1, & i-j=0, \\
a_{i}, & j=i+1, \\
b_{i}, & j=i+2,
\end{array} \quad 0 \leq i \leq N-1,0 \leq j \leq N .\right.
$$

To derive a formula for the derivative of the modal basis functions, we first prove the following result.

Lemma 2 The operational matrix for derivative of the DBPs, $\mathbf{P}$ satisfies

$$
\tilde{\boldsymbol{\Psi}}^{\prime}=\mathbf{P} \tilde{\boldsymbol{\Psi}}
$$

where the matrix $\mathbf{P}=\left[p_{i, j}: 0 \leq i, j \leq N\right]$ is given by

$$
p_{i, j}= \begin{cases}-(-1)^{i}(N+1)\left(\begin{array}{c}
N+1 \\
i+1
\end{array}\right)+N \delta_{i, 0}+\delta_{i, 1}, & j=0, \\
-p_{N-i, 0} & j=N, \\
i, & j=i-1, j \neq 0, \\
N-2 i, & j=i, j \neq 0, N \\
-N+i, & j=i+1, j \neq N\end{cases}
$$

Proof The DBPs $\tilde{\boldsymbol{\Psi}}$ is a basis for $P_{N}$, so we expand $\tilde{\psi}_{i}^{\prime}(x)$ for $0 \leq i \leq N$, as

$$
\tilde{\psi}_{i}^{\prime}(x)=\sum_{j=0}^{N} p_{i, j} \tilde{\psi}_{j}(x) .
$$

Integration by parts and 2.3 imply that

$$
\begin{aligned}
p_{i, j} & =\int_{0}^{1} \tilde{\psi}_{i}^{\prime}(x) \phi_{j}(x) d x \\
& =\tilde{\psi}_{i}(1) \delta_{j, N}-\tilde{\psi}_{i}(0) \delta_{j, 0}-\int_{0}^{1} \tilde{\psi}_{i}(x)\left((N-j+1) \phi_{j-1}(x)-(N-2 j) \phi_{j}(x)-(j+1) \phi_{j+1}(x)\right) d x .
\end{aligned}
$$

The biorthogonality 2.6 gives

$$
p_{i, j}=\tilde{\psi}_{i}(1) \delta_{j, N}-\tilde{\psi}_{i}(0) \delta_{j, 0}-\left((N-j+1) \delta_{i, j-1}-(N-2 j) \delta_{i, j}-(j+1) \delta_{i, j+1}\right) .
$$

Now, the result is proved by considering 2.10 and 2.11.

Remark 1 The matrix $\mathbf{P}$ is a sparse matrix of order $N+1$ with $p_{i, j}=0$ for $|i-j|>1, j \neq 0, N$; for instance, see the matrix given below.

Corollary 1 Set $\alpha_{i, 0}=-(-1)^{i}(N+1)\left(\begin{array}{c}N+1 \\ i+1\end{array}\right)+N \delta_{i, 0}+\delta_{i, 1}$ for $0 \leq i \leq N$. Then, from 2.15), we infer the following five-term recurrence relation is deduced

$$
\begin{aligned}
\tilde{\psi}_{i}^{\prime}(x) & =\alpha_{i, 0} \tilde{\psi}_{0}(x)+\left(1-\delta_{i, 1}\right) i \tilde{\psi}_{i-1}(x)+\left(1-\delta_{i, 0}\right)\left(1-\delta_{i, N}\right)(N-2 i) \tilde{\psi}_{i}(x) \\
& -\left(1-\delta_{i, N-1}\right)(N-i) \tilde{\psi}_{i+1}(x)-\alpha_{N-i, 0} \tilde{\psi}_{N}(x),
\end{aligned}
$$

where we set $\tilde{\psi}_{i} \equiv 0$ for $i<0$ and $i>N$. 
We derive the transformation matrices that map the Bernstein and Chebyshev coefficients

Now we derive the transformation matrix that maps the derivative of modal basis functions to DBPs. This facilitates the use of Galerkin method in the next section. In the following, $(p, q)-$ band matrix stands for a matrix with $p$ and $q$ nonzero diagonals below and above the main diagonal, respectively.

Lemma 3 Let the vectors $\boldsymbol{\Psi}$ and $\tilde{\boldsymbol{\Psi}}$ be defined as in 2.12) and 2.13), respectively. Then,

$$
\Psi^{\prime}=\mathbf{Q} \tilde{\Psi}
$$

where $\mathbf{Q}$ is an $(N-1) \times(N+1),(1,3)$ - band matrix given by $\mathbf{Q}=\mathbf{G P}$.

Proof Combining 2.14 with 2.15, implies $\mathbf{Q}=\mathbf{G P}$. To prove that $\mathbf{Q}$ is a $(1,3)-$ band matrix, it is sufficient to show that $q_{i, 0}=0$ for $i>1$ and $q_{i, N}=0$ for $i<N-2$,

$$
\begin{aligned}
q_{i, 0} & =(\mathbf{G P})_{i, 0}=p_{i, 0}+a_{i} p_{i+1,0}+b_{i} p_{i+2,0} \\
& =-\left(\tilde{\psi}_{i}(0)+a_{i} \tilde{\psi}_{i+1}(0)+b_{i} \tilde{\psi}_{i+2}(0)\right)=-\psi_{i}(0)=0,
\end{aligned}
$$

and for $i<N-2$, by (1)

$$
\begin{aligned}
q_{i, N} & =p_{i, N}+a_{i} p_{i+1, N}+b_{i} p_{i+2, N}=\tilde{\psi}_{N-i}(0)+a_{i} \tilde{\psi}_{N-i-1}(0)+b_{i} \tilde{\psi}_{N-i-2}(0) \\
& =\tilde{\psi}_{i}(1)+a_{i} \tilde{\psi}_{i+1}(1)+b_{i} \tilde{\psi}_{i+2}(1)=\psi_{i}(1)=0 .
\end{aligned}
$$

Note that $\psi_{i}$ 's vanish at the boundary values according to Proposition 1 . The proof is complete.

To see the sparsity of the transformation matrices, $\mathbf{P}, \mathbf{G}$ and $\mathbf{Q}$ for $N=6$ are shown in the following.

$$
\mathbf{G}=\left[\begin{array}{lllllll}
1 & 4 & \frac{1}{7} & 0 & 0 & 0 & 0 \\
0 & 1 & 1 & \frac{2}{5} & 0 & 0 & 0 \\
0 & 0 & 1 & \frac{8}{5} & 1 & 0 & 0 \\
0 & 0 & 0 & 1 & \frac{5}{2} & \frac{5}{2} & 0 \\
0 & 0 & 0 & 0 & 1 & 4 & 7
\end{array}\right], \quad \mathbf{P}=\left[\begin{array}{cccccccc}
-43 & -6 & 0 & 0 & 0 & 0 & 7 \\
148 & 4 & -5 & 0 & 0 & 0 & -49 \\
-245 & 2 & 2 & -4 & 0 & 0 & 147 \\
245 & 0 & 3 & 0 & -3 & 0 & -245 \\
-147 & 0 & 0 & 4 & -2 & -2 & 245 \\
49 & 0 & 0 & 0 & 5 & -4 & -148 \\
-7 & 0 & 0 & 0 & 0 & 6 & 43
\end{array}\right], \quad \mathbf{Q}=\left[\begin{array}{ccccccc}
\frac{46}{7} & -\frac{24}{7} & -\frac{18}{7} & -\frac{4}{7} & 0 & 0 & 0 \\
1 & 6 & -\frac{9}{5} & -4 & -\frac{6}{5} & 0 & 0 \\
0 & 2 & \frac{34}{5} & 0 & -\frac{34}{5} & -2 & 0 \\
0 & 0 & 3 & 10 & \frac{9}{2} & -15 & -\frac{5}{2} \\
0 & 0 & 0 & 4 & 18 & 24 & -46
\end{array}\right]
$$

\section{Variational formulation of the problem (1.1) and the spectral discretization}

In this section, at first the problem (1.1)-(1.3) is discretized in time. Then we develop a matrix approach Bernstein dual-Petrov-Galerkin method using the results of the previous section.

\subsection{Time discretization}

Consider the subdiffusion equation (1.1) at $t=t_{k+1}, k \geq 0$ as

$$
D_{t}^{\alpha} u\left(x, y, t_{k+1}\right)=\kappa \Delta u\left(x, y, t_{k+1}\right)+S\left(x, y, t_{k+1}\right) .
$$


Let $u^{k}$ be an approximation of $u$ at $t=t_{k}=k \tau$ for $k=0,1, . ., M$, where $\tau=\frac{T}{M}$ is the time step length. The time fractional derivative can be approximated by definition (1.4) and using forward difference for the derivative inside as

$$
D_{t}^{\alpha} u\left(x, y, t_{k+1}\right)=\mu\left(u^{k+1}-\left(1-b_{1}\right) u^{k}-\sum_{j=1}^{k-1}\left(b_{j}-b_{j+1}\right) u^{k-j}-b_{k} u^{0}\right)+r_{\tau}^{k+1}, \quad k \geq 1,
$$

where $\mu=\frac{1}{\tau^{\alpha} \Gamma(2-\alpha)}$ and $b_{j}=(j+1)^{1-\alpha}-j^{1-\alpha}$ for $k \geq 0$ and $0 \leq j \leq k$. The error is bounded by

$$
\left|r_{\tau}^{k+1}\right| \leq \tilde{c}_{u} \tau^{2-\alpha}
$$

where the coefficient $\tilde{c}_{u}$ depends only on $u$ [4. The time discretization $(3.2)$ is referred to as L1 approximation (see e.g. [4, 26]). Substituting from (3.2) into (3.1) and multiplying both sides by $\tau^{\alpha} \Gamma(2-\alpha)$ and dropping $(x, y)$, the following time-discrete scheme is obtained

$$
\begin{aligned}
& u^{k+1}-\alpha_{0} \Delta u^{k+1}=f^{k+1}, \quad k \geq 0, \\
& f^{k+1}:=\left(1-b_{1}\right) u^{k}+\sum_{j=1}^{k-1}\left(b_{j}-b_{j+1}\right) u^{k-j}+b_{k} u^{0}+\frac{1}{\mu} S^{k+1},
\end{aligned}
$$

with $\alpha_{0}=\frac{k}{\mu}$ and $u^{0}=g$ is given by the initial condition 1.2 with the error

$$
r^{k+1} \leq \tau^{\alpha} \Gamma(2-\alpha)\left|r_{\tau}^{k+1}\right| \leq \tilde{c}_{u} \tau^{2}
$$

For $k=0$, it reads as

$$
u^{1}-\alpha_{0} \kappa \Delta u^{1}=\left(1-b_{1}\right) u^{1}+b_{1} u^{0}+\frac{1}{\mu} S^{1} .
$$

The boundary conditions for the semidiscrete problem is $u^{k+1}=0$ on $\partial \Omega$.

\subsection{Weak and spectral formulation}

Consider the problem 3.4 with $\Omega=I^{2}, I=(0,1)$ and the homogeneous Dirichlet boundary conditions $\left.u^{k+1}\right|_{\partial \Omega}=0$. We seek an approximate solution in the Sobolev space $H_{0}^{1}(\Omega)=\left\{u \in H^{1}(\Omega), u=\right.$ 0 , on $\partial \Omega\}$. A weak formulation of the problem $(3.4)$ is to find $u^{k+1} \in H_{0}^{1}(\Omega)$ such that $\forall v \in H_{0}^{1}(\Omega)$ :

$$
\left(u^{k+1}, v\right)+\alpha_{0}\left(\nabla u^{k+1}, \nabla v\right)=\left(\left(1-b_{1}\right) u^{k}+\sum_{j=1}^{k-1}\left(b_{j}-b_{j+1}\right) u^{k-j}+b_{k} u^{0}+\frac{1}{\mu} S^{k+1}, v\right) .
$$

Let $\mathbb{P}_{N}$ be the space of polynomials over $I$ with degree not exceeding $N$ and $\left(\mathbb{P}_{N}^{0}\right)^{2}=\left\{v \in\left(\mathbb{P}_{N}\right)^{2}\right.$ : $v=0$, on $\partial \Omega\}$.

The Galerkin formulation of the 3.7 is to find $u_{N}^{k+1} \in\left(\mathbb{P}_{N}^{0}\right)^{2}$ such that $\forall v_{N} \in\left(\mathbb{P}_{N}^{0}\right)^{2}$ :

$$
\left(u_{N}^{k+1}, v_{N}\right)+\alpha_{0}\left(\nabla u_{N}^{k+1}, \nabla v_{N}\right)=\left(\left(1-b_{1}\right) u_{N}^{k}+\sum_{j=1}^{k-1}\left(b_{j}-b_{j+1}\right) u_{N}^{k-j}+b_{k} u_{N}^{0}+\frac{1}{\mu} I_{N} S^{k+1}, v_{N}\right)
$$

with $(f, g)$ being the standard inner product and $I_{N}$ an interpolation operator. 


\subsubsection{Bernstein dual-Petrov-Galerkin method}

Since $\operatorname{dim} \mathbb{P}_{N}^{0}=N-1$, and due to 2.1 , we choose a basis for it by removing the first and last Bernstein polynomials of degree $N$, i.e.,

$$
\mathbf{\Phi}=\left[\phi_{i}(x): 1 \leq i \leq N-1\right]^{T} .
$$

Using (2.3), it is easy to verify

$$
\Phi^{\prime}=\mathbf{D} \Phi+\mathbf{d}
$$

where $\mathbf{D}=\operatorname{tridiag}(N-i+1,2 i-N,-(i+1))$ is a tridiagonal matrix of order $N-1$ and $\mathbf{d}=$ $N\left[\phi_{0}, 0, \ldots, 0,-\phi_{N}\right]^{T}$ is an $(N-1)$-vector.

Assuming $N_{x}=N_{y}=N$, we use the tensor product of the basis functions of $\mathbb{P}_{0}^{N}$ as a basis for two dimensional case, $\left\{\phi_{i}(x) \phi_{j}(y): 1 \leq i, j \leq N-1\right\}$ and consider an approximate solution of (3.4) as

$$
u_{N}^{k+1}=\sum_{i, j=1}^{N-1} \hat{u}_{i, j}^{k+1} \phi_{i}(x) \phi_{j}(y)=\boldsymbol{\Phi}^{T}(x) \mathbf{U}^{k+1} \boldsymbol{\Phi}(y), \quad(x, y) \in \Omega,
$$

where $\boldsymbol{\Phi}(\cdot)=\left[\phi_{i}(\cdot): 1 \leq i \leq N-1\right]^{T}$ and $\mathbf{U}^{k+1}=\left[\hat{u}_{i, j}^{k+1}\right]$. Let us use the following notations.

$$
\begin{aligned}
& a_{i, j}=\int_{I} \phi_{j}^{\prime}(x) \psi_{i}^{\prime}(x) d x, \quad \mathbf{A}=\left[a_{i, j}\right], \\
& b_{i, j}=\int_{I} \phi_{j}(x) \psi_{i}(x) d x, \quad \mathbf{B}=\left[b_{i, j}\right], \\
& f_{i, j}^{k+1}=\int_{\Omega} I_{N} f^{k+1}(x, y) \psi_{j}(x) \psi_{i}(y) d \Omega,
\end{aligned}
$$

for $1 \leq j \leq N-1,0 \leq i \leq N-2$.

Taking the test functions of (3.8) as $v=\psi_{l}(x) \psi_{m}(y)$ for $l, m=0,1, \ldots, N-2$, it is seen that the spectral form 3.8 is equivalent to the following linear system:

$$
\mu_{\tau}^{\alpha} \mathbf{B} \mathbf{U}^{k+1} \mathbf{B}^{T}+\kappa\left(\mathbf{A} \mathbf{U}^{k+1} \mathbf{B}^{T}+\mathbf{B} \mathbf{U}^{k+1} \mathbf{A}^{T}\right)=\mathbf{F}^{k+1}, \quad k \geq 0,
$$

that can be equivalently written as a Sylvester equation but it requires computing the inverse of $\mathbf{B}$. Although $\mathbf{B}$ has only three nonzero diagonals, it can be shown that its inverse is a dense matrix and so we avoid transforming to Sylvester equation. Instead we use the equivalent tensor product form

$$
\left(\mu_{\tau}^{\alpha} \mathbf{B} \otimes \mathbf{B}+\kappa(\mathbf{B} \otimes \mathbf{A}+\mathbf{A} \otimes \mathbf{B})\right) \mathbf{u}^{k+1}=\mathbf{f}^{k+1},
$$

with $\mathbf{f}=\left[f_{1,0}, \ldots, f_{q, 0} ; f_{1,1} \ldots, f_{q, 1} ; \ldots ; f_{1, q-1, \ldots, f_{q, q-1}}\right]^{T}, q=N-1$. It is worth to note that the coefficient matrix of the linear system (3.13) is the same for all time steps so it is to be evaluated just once for all $k \geq 0$.

In terms of the trial vector (3.9), and test vector 2.13, we may write

$$
\mathbf{A}=\int_{I} \boldsymbol{\Psi}^{\prime} \boldsymbol{\Phi}^{\prime T} d x, \quad \mathbf{B}=\int_{I} \boldsymbol{\Psi} \boldsymbol{\Phi}^{T}
$$


To facilitate the computations, in what follows, these matrices are related to the transformation matrices introduced in Section 2.2. First, note that by the biorthogonality (2.6), we have

$$
\int_{I} \tilde{\boldsymbol{\Psi}} \boldsymbol{\Phi}^{T} d x=\left[\begin{array}{ccccc}
0 & 0 & \cdots & 0 & 0 \\
1 & 0 & \cdots & 0 & 0 \\
0 & 1 & \cdots & 0 & 0 \\
\vdots & \vdots & \ddots & \vdots & \vdots \\
0 & 0 & \cdots & 1 & 0 \\
0 & 0 & \cdots & 0 & 1 \\
0 & 0 & \cdots & 0 & 0
\end{array}\right]=: \tilde{\mathbf{I}}
$$

Now from (2.14), and writing $\mathbf{G}$ as $\mathbf{G}=\left[\mathbf{g}_{0}, \mathbf{g}_{1}, \ldots, \mathbf{g}_{N}\right]$, we get

$$
\mathbf{B}=\int_{I} \mathbf{G} \tilde{\boldsymbol{\Psi}} \boldsymbol{\Phi}^{T} d x=\mathbf{G} \tilde{\mathbf{I}}=\left[\mathbf{g}_{1}, \mathbf{g}_{2}, \ldots, \mathbf{g}_{N-1}\right] .
$$

So $\mathbf{B}$ is a tridiagonal matrix whose entries are given by

$$
b_{i, j}= \begin{cases}1, & j=i+1 \\ a_{i}, & j=i, \\ b_{i}, & j=i-1 \\ 0, & \text { otherwise }\end{cases}
$$

where $a_{i}$ 's and $b_{i}$ 's are easily computed by 2.9. On the other hand, from Lemma 3 the Bernstein operational matrix of differentiation 3.10 and $(3.14)$, we obtain

$$
\begin{aligned}
\mathbf{A} & =\int_{I} \mathbf{Q} \tilde{\mathbf{\Psi}}\left(\boldsymbol{\Phi}^{\mathbf{T}} \mathbf{D}^{\mathbf{T}}+\mathbf{d}^{\mathbf{T}}\right) d x \\
& =\mathbf{Q} \tilde{\mathbf{I}} \mathbf{D}^{\mathbf{T}}+N\left[\mathbf{q}_{0}, \mathbf{0}, \ldots, \mathbf{0}, \mathbf{q}_{N}\right] \\
& =\left[\mathbf{q}_{1}, \ldots, \mathbf{q}_{N-1}\right] \mathbf{D}^{T}+N\left[\mathbf{q}_{0}, \mathbf{0}, \ldots, \mathbf{0}, \mathbf{q}_{N}\right],
\end{aligned}
$$

where $\mathbf{Q}=\left[\mathbf{q}_{0}, \mathbf{q}_{1}, \ldots, \mathbf{q}_{N}\right]$ is a $(1,3)$ - band matrix introduced Lemma 3 . Hence, Q $\tilde{\mathbf{I}}$ is a pentadiagonal matrix and $\mathbf{A}$ is the product of a pentadiagonal and a tridiagonal matrix plus a sparse matrix. From Lemma 3 and (3.16), it is seen that $\mathbf{A}$ is a seven-diagonal matrix.

Notice that the solution of linear system (3.13) requires the matrices $\mathbf{A}$ and $\mathbf{B}$. $\mathbf{A}$ is obtained by a sparse matrix-matrix multiplication $(3.16)$ and entries of $\mathbf{B}$ are given by $(3.15)$.

Remark 2 Since the coefficient matrix of the linear system 3.13 remains intact for a fixed $\tau$, only the RHS vector to be computed for different time steps, $k=0,1, \ldots$ up to a desired time. So it is efficient to use a band-LU factorization for solving the system. It is remarkable that for a $(2 p+1)-$ band matrix, the LU-factorization can be done with $O\left(N p^{2}\right)$ flops and backward substitutions require $O(N p)$ flops [16. Section 4.3].

\section{Stability and convergence analysis}

For the error analysis, we assume the problem (1.1) to be homogeneous, $S=0$.

For $\alpha \geq 0$, the bilinear form $a(u, v)=(\nabla u, \nabla v)+\alpha(u, v)$ in $(3.8)$ is continuous and coercive in $H_{0}^{1}(\Omega) \times \bar{H}_{0}^{1}(\Omega)$. The existence and uniqueness of the solution for both the weak form 3.8 and the Galerkin form (3.8) is guarantied by the well-known Lax-Milgram lemma. 
We define the following inner product and the associated energy norm on $H_{0}^{1}(\Omega)$ :

$$
(u, v)=\int_{\Omega} u v d \Omega, \quad(u, v)_{1}=(u, v)+\alpha_{0}(\nabla u, \nabla v), \quad\|u\|_{1}=(u, u)_{1}^{\frac{1}{2}} .
$$

Theorem 1 The weak form (3.7) is unconditionally stable:

$$
\left\|u^{k}\right\|_{1} \leq\left\|u^{0}\right\|, \quad k=1, \ldots, M
$$

Proof Let $v=u^{1}$ in 3.7 . Then,

$$
\left(u^{1}, u^{1}\right)+\alpha_{0}\left(\nabla u^{1}, \nabla u^{1}\right)=\left(u^{0}, u^{1}\right)
$$

giving (4.2) for $k=1$, by the definition (4.1), the Schwarz inequality and the inequality $\|v\| \leq\|v\|_{1}$. By mathematical induction, assume 4.2 holds for $k=0, \ldots, n$. Let $v=u^{n+1}$ in (3.7), i.e.,

$$
\begin{gathered}
\left(u^{n+1}, u^{n+1}\right)+\alpha_{0}\left(\nabla u^{n+1}, \nabla u^{n+1}\right)=\left(1-b_{1}\right)\left(u^{n}, u^{n+1}\right) \\
+\sum_{j=1}^{n-1}\left(b_{j}-b_{j+1}\right)\left(u^{n-j}, u^{n+1}\right)+b_{n}\left(u^{0}, u^{n+1}\right) .
\end{gathered}
$$

It is easy to see that the RHS coefficients in (3.4) are positive. So we obtain

$$
\begin{aligned}
\left\|u^{n+1}\right\|_{1} & \leq\left(1-b_{1}\right)\left\|u^{n}\right\|+\sum_{j=1}^{n-1}\left(b_{j}-b_{j+1}\right)\left\|u^{n-j}\right\|+b_{n}\left\|u^{0}\right\| \\
& \leq\left(\left(1-b_{1}\right)+\sum_{j=1}^{n-1}\left(b_{j}-b_{j+1}\right)+b_{n}\right)\left\|u^{0}\right\|=\left\|u^{0}\right\| .
\end{aligned}
$$

So the proof is done.

Theorem 2 Let $u$ be the solution of the equation (1.1) with conditions (1.2)- (1.3) and $u^{k}$ be the solution of the the semidiscrete problem (3.4). Then,

$$
\begin{array}{ll}
\left\|u\left(t_{k}\right)-u^{k}\right\|_{1} \leq \frac{c_{u}}{1-\alpha} T^{\alpha} \tau^{2-\alpha}, & 0<\alpha<1, \\
\left\|u\left(t_{k}\right)-u^{k}\right\|_{1} \leq c_{u} T \tau, & \text { as } \alpha \rightarrow 1 .
\end{array}
$$

Proof The idea of the proof comes from [20]. We first prove

$$
\left\|u\left(t_{k}\right)-u^{k}\right\|_{1} \leq \frac{c_{u}}{b_{k-1}} \tau^{2}, \quad k=1, \ldots, M
$$

By (1.1) and (3.6), we have

$$
\left(e^{1}, v\right)+\alpha_{0}\left(\nabla e^{1}, \nabla v\right)=\left(e^{0}, v\right)+\left(r^{1}, v\right), \quad \forall v \in H_{0}^{1}(\Omega),
$$

in which $e^{k}:=u\left(t_{k}\right)-u^{k}$. For $v=e^{1}$ and by using $e^{0}=0,\|v\| \leq\|v\|_{1}$ and (3.5), we get

$$
\left\|e^{1}\right\|_{1} \leq c_{u} \tau^{2}
$$


i.e., 4.5 holds for $k=1$. By induction, assume (4.5) holds for $k \leq n$. Using (1.1) and (3.4), we get

$$
\begin{aligned}
\left(e^{n+1}, v\right)+\alpha_{1}\left(\nabla e^{n+1}, \nabla v\right)=\left(1-b_{1}\right)\left(e^{n}, v\right) & \\
+ & \sum_{j=1}^{n-1}\left(b_{j}-b_{j+1}\right)\left(e^{n-j}, v\right)+b_{n}\left(e^{0}, v\right)+\left(r^{n+1}, v\right), \quad \forall v \in H_{0}^{1}(\Omega) .
\end{aligned}
$$

For $v=e^{n+1}$, it reads as

$$
\begin{aligned}
\left\|e^{n+1}\right\|_{1}^{2} & \leq\left(1-b_{1}\right)\left\|e^{n}\right\|\left\|e^{n+1}\right\|_{1}+\sum_{j=1}^{n-1}\left(b_{j}-b_{j+1}\right)\left\|e^{n-j}\right\|\left\|e^{n+1}\right\|_{1}+\left\|r^{n+1}\right\|\left\|e^{n+1}\right\|_{1}, \\
\Rightarrow\left\|e^{n+1}\right\|_{1} & \leq\left(1-b_{1}\right) \frac{c_{u}}{b_{n-1}} \tau^{2}+\sum_{j=1}^{n-1}\left(b_{j}-b_{j+1}\right) \frac{c_{u}}{b_{n-j-1}} \tau^{2}+c_{u} \tau^{2} \\
& \leq\left(\left(1-b_{1}\right)+\sum_{j=1}^{n-1}\left(b_{j}-b_{j+1}\right)+b_{n}\right) \frac{c_{u}}{b_{n}} \tau^{2}=\frac{c_{u}}{b_{n}} \tau^{2},
\end{aligned}
$$

proving (4.5) for $k=n+1$ that completes the proof of 4.5).

Consider $f(t)=t^{1-\alpha}$, then there exists a $\xi, k-1<\xi<k \leq M$ such that

$$
b_{k-1} \tau^{-\alpha}=\frac{(k \tau)^{1-\alpha}-(\tau(k-1))^{1-\alpha}}{\tau}=(1-\alpha)(\xi \tau)^{-\alpha} \geq(1-\alpha)(k \tau)^{-\alpha} \geq(1-\alpha)(T)^{-\alpha},
$$

which gives

$$
\frac{c_{u}}{b_{k-1}} \tau^{2} \leq \frac{c_{u}}{1-\alpha} T^{\alpha} \tau^{2-\alpha}
$$

Now using this along with 4.5 proves 4.3 .

In order to derive 4.4 , we first prove

$$
\left\|u\left(t_{k}\right)-u^{k}\right\|_{1} \leq c_{u} k \tau^{2}, \quad k=1, \ldots, M .
$$

By (4.6), the inequality 4.8 holds for $k=1$. Assume 4.8 holds for $k=1, \ldots, n, n \leq M-1$. Then, from (1.1), 3.4 and (3.5), we obtain

$$
\begin{aligned}
\left\|e^{n+1}\right\|_{1} & \leq\left(1-b_{1}\right)\left\|e^{n}\right\|+\sum_{j=1}^{n-1}\left(b_{j}-b_{j+1}\right)\left\|e^{n-j}\right\|+\left\|r^{n+1}\right\| \\
& \leq\left(\left(1-b_{1}\right) \frac{n}{n+1}+\sum_{j=1}^{n-1}\left(b_{j}-b_{j+1}\right) \frac{n-j}{n+1}+\frac{1}{(n+1)}\right) c_{u}(n+1) \tau^{2} \\
& \leq\left(\left(1-b_{1}\right) \frac{n}{n+1}+\left(b_{1}-b_{n}\right) \frac{n}{n+1}-\left(b_{1}-b_{n}\right) \frac{1}{n+1}+\frac{1}{(n+1)}\right) c_{u}(n+1) \tau^{2} \\
& =\left(1-b_{n} \frac{n}{n+1}-\left(b_{1}-b_{n}\right) \frac{1}{n+1}\right) c_{u}(n+1) \tau^{2} \leq c_{u}(n+1) \tau^{2} .
\end{aligned}
$$

So 4.8 holds for $k=n+1$. From $k \tau \leq T$ and 4.8, we get 4.4. 
4.1 Convergence of the full discretization scheme

Let $\pi_{N}^{1,0}$ be the $H^{1}$-orthogonal projection operator from $H_{0}^{1}(\Omega)$ into $\left(\mathbb{P}_{N}^{0}\right)^{2}$ associated with the energy norm $\|\cdot\|_{1}$ defined in 4.1]. Due to the equivalence of this norm with the standard $H^{1}$ norm, we have the following error estimation [20, Relation (4.3)]

$$
\left\|u-\pi_{N}^{1,0} u\right\|_{1} \leq c N^{1-m}\|u\|_{m}, \quad u \in H_{0}^{m}(\Omega) \cap H_{0}^{1}(\Omega), m \geq 1 .
$$

The idea of the proof for the following result comes from the paper [20].

Theorem 3 Let $u^{k}, k=0, \ldots, M$ be the solution of the variational formulation 3.7 and $u_{N}^{k}$ be the solution of the scheme (3.8), assuming $u^{0}=\pi_{N}^{1,0} u^{0}$ and $u^{k} \in H^{m}(\Omega) \cap H_{0}^{1}(\Omega)$ for some $m>1$. Then,

$$
\begin{aligned}
& \left\|u^{k}-u_{N}^{k}\right\|_{1} \leq \frac{c}{1-\alpha} \tau^{-\alpha} N^{1-m} \max _{0 \leq j \leq k}\left\|u^{j}\right\|_{m}, \quad 0<\alpha<1, \\
& \left\|u^{k}-u_{N}^{k}\right\|_{1} \leq c N^{1-m} \sum_{j=0}^{k}\left\|u^{j}\right\|_{m}, \quad \alpha \rightarrow 1,
\end{aligned}
$$

for $k=1, \ldots, M$, where $c$ depends only on $T^{\alpha}$.

Proof We have $\left(u^{k+1}-\pi_{N}^{1,0} u^{k+1}, v_{N}\right)_{1}=0, \forall v_{N} \in\left(\mathbb{P}_{N}^{0}\right)^{2}$ by the projection operator. By definition of the norm 4.1, we get

$$
\left(\pi_{N}^{1,0} u^{k+1}, v_{N}\right)+\alpha_{1}\left(\nabla \pi_{N}^{1,0} u^{k+1}, \nabla v_{N}\right)=\left(u^{k+1}, v_{N}\right)+\alpha_{1}\left(\nabla u^{k+1}, \nabla v_{N}\right), \quad \forall v_{N} \in\left(\mathbb{P}_{N}^{0}\right)^{2}
$$

By the weak form (3.7), the RHS of the above equation is replaced as

$$
\begin{aligned}
\left(\pi_{N}^{1,0} u^{k+1}, v_{N}\right)+\alpha_{1}\left(\nabla \pi_{N}^{1,0} u^{k+1}, \nabla v_{N}\right)=\left(1-b_{1}\right)\left(u^{k}, v_{N}\right) & \\
& +\sum_{j=1}^{k-1}\left(b_{j}-b_{j+1}\right)\left(u^{k-j}, v_{N}\right)+b_{k}\left(u^{0}, v_{N}\right), \quad \forall v_{N} \in\left(\mathbb{P}_{N}^{0}\right)^{2}
\end{aligned}
$$

Subtracting 4.11 from 3.8, we have

$$
\begin{aligned}
& \left(\tilde{e}_{N}^{k+1}, v_{N}\right)+\alpha_{1}\left(\frac{\partial \tilde{e}_{N}^{k+1}}{\partial x}, \frac{\partial v_{N}}{\partial x}\right)=\left(1-b_{1}\right)\left(e_{N}^{k}, v_{N}\right) \\
& +\sum_{j=1}^{k-1}\left(b_{j}-b_{j+1}\right)\left(e_{N}^{k-j}, v_{N}\right)+b_{k}\left(e_{N}^{0}, v_{N}\right), \quad \forall v_{N} \in\left(\mathbb{P}_{N}^{0}\right)^{2}
\end{aligned}
$$

where $e_{N}^{k+1}=u^{k+1}-u_{N}^{k+1}$ and $\tilde{e}_{N}^{k+1}=\pi_{N}^{1,0} u^{k+1}-u_{N}^{k+1}$. Let $v_{N}=\tilde{e}_{N}^{k+1}$, then

$$
\left\|\tilde{e}_{N}^{k+1}\right\|_{1} \leq\left(1-b_{1}\right)\left\|e_{N}^{k}\right\|+\sum_{j=1}^{k-1}\left(b_{j}-b_{j+1}\right)\left\|e_{N}^{k-j}\right\|+b_{k}\left\|e_{N}^{0}\right\|
$$

With $\left\|e_{N}^{k+1}\right\|_{1} \leq\left\|\tilde{e}_{N}^{k+1}\right\|_{1}+\left\|u^{k+1}-\pi_{N}^{1,0} u^{k+1}\right\|_{1}$, we obtain

$$
\left\|e_{N}^{k+1}\right\|_{1} \leq\left(1-b_{1}\right)\left\|e_{N}^{k}\right\|+\sum_{j=1}^{k-1}\left(b_{j}-b_{j+1}\right)\left\|e_{N}^{k-j}\right\|+b_{k}\left\|e_{N}^{0}\right\|+c N^{1-m}\left\|u^{k+1}\right\| .
$$


As in the proof of Theorem 2 it is first proved by induction that:

$$
\begin{aligned}
& \left\|e_{N}^{k+1}\right\|_{1} \leq \frac{1}{b_{k-1}} \max _{0 \leq j \leq k}\left\|u^{j}-\pi_{N}^{1,0} u^{j}\right\|_{1}, \quad 0<\alpha<1, \\
& \left\|e_{N}^{k+1}\right\|_{1} \leq \sum_{j=0}^{k}\left\|u^{j}-\pi_{N}^{1,0} u^{j}\right\|_{1}, \quad \alpha \rightarrow 1,
\end{aligned}
$$

for $0 \leq k \leq M$. Then, by using (4.7) and the projection error 4.9 the desired result is derived.

The following theorem is obtained by the triangle inequality $\left\|u\left(\cdot, t_{k}\right)-u_{N}^{k}\right\|_{1} \leq\left\|u\left(\cdot, t_{k}\right)-u^{k}\right\|_{1}+$ $\left\|u^{k}-u_{N}^{k}\right\|_{1}$ along with the inequalities 4.3 and 4.10.

Theorem 4 Let $u$ be the solution of the problem 1.1) with the initial and boundary conditions given by (1.2)-(1.3) and $u_{N}^{k}$ be the solution of the scheme 3.8). Then, assuming $u_{N}^{0}=\pi_{N}^{1,0} u^{0}$ and $u \in H^{m}(\Omega) \cap$ $H_{0,}^{1}(\Omega)$, we have

$$
\begin{aligned}
& \left\|u\left(t_{k}\right)-u_{N}^{k}\right\|_{1} \leq \frac{C T^{\alpha}}{1-\alpha}\left(c_{u} \tau^{2-\alpha}+c \tau^{-\alpha} N^{1-m} \sup _{0<t<T}\|u(x, t)\|_{m}\right), \quad k \leq M, \quad 0<\alpha<1, \\
& \left\|u\left(t_{k}\right)-u_{N}^{k}\right\|_{1} \leq T^{\alpha}\left(c_{u} \tau+c \tau^{-1} N^{1-m} \sup _{0<t<T}\|u(x, t)\|_{m}\right), \quad k \leq M, \quad \alpha \rightarrow 1 .
\end{aligned}
$$

The constants $C$ and $c$ are independent of $\tau, T, N$.

It is seen that the method has the so-called spectral convergence in space and the order of convergence $O\left(\tau^{2-\alpha}\right)$ in time.

\section{Numerical examples}

Here, some numerical experiments are provided to show the accuracy of the proposed method. For the computations, we use Maple 18. on a laptop with CPU core i3 $1.9 \mathrm{GHz}$ and RAM 4 running Windows 8.1 platform. To compute the errors, we use the discrete $L^{2}$ and $L^{\infty}$ errors defined as

$$
\begin{aligned}
L^{2} & \approx\left(\frac{1}{\mathcal{N}^{2}} \sum_{i, j=0}^{\mathcal{N}-1}\left|u\left(x_{i}, y_{j}, t_{m}\right)-u_{N}^{m}\left(x_{i}, y_{j}\right)\right|^{2}\right)^{1 / 2} \\
L^{\infty} & \approx \max _{0 \leq i, j \leq \mathcal{N}}\left|u\left(x_{i}, y_{j}, t_{m}\right)-u_{N}^{m}\left(x_{i}, y_{j}\right)\right|,
\end{aligned}
$$

respectively, where $u$ is the exact solution of the problem $1.1-(1.3), u_{N}^{m}$ is the approximation solution 3.11 at $t=t_{m}=m \tau, x_{i}=\frac{i}{\mathcal{N}}, y_{j}=\frac{j}{N}$ and $\mathcal{N}=100$. Also, the convergence rates in space and time are respectively computed by

$$
\operatorname{rate}_{N_{i}}=\frac{\log \frac{E\left(N_{i}, \tau\right)}{E\left(N_{i-1}, \tau\right)}}{\log \frac{N_{i-1}}{N_{i}}}, \quad \operatorname{rate}_{\tau_{i}}=\frac{\log \frac{E\left(N, \tau_{i}\right)}{E\left(N, \tau_{i-1}\right)}}{\log \frac{\tau_{i}}{\tau_{i-1}}}
$$

where $E(h, \tau)$ is the error with $h=1 / N$ where $N$ stands for the dimension of basis and $\tau$ is the time-step size. However, as it is common in the literature, we will show the spectral convergence of the proposed method by logarithmic scaled error plots.

It is worth to mention that as we derived the operational matrices in Section 2.2 with special structures, the proposed method finally leads to the linear system 3.13 that is sparse and banded. 

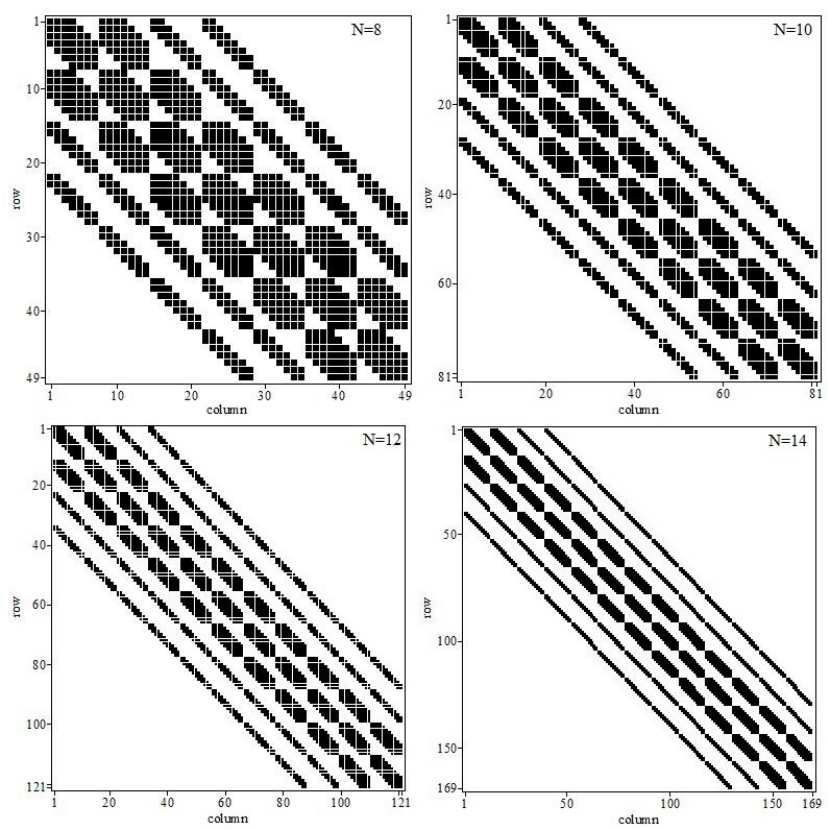

Fig. 5.1 The coefficient matrix of the linear system 3.13.

To see the sparsity and bandedness, the nonzero entries of the coefficinet matrix of (3.13) are depicted in Fig. 5.1 using sparsematrixplot command of Maple. It is seen that the density decreases rapidly as $N$ increases.

Example 1 Consider the problem 1.1 with $\kappa=1$ and the exact solution $u(x, y, t)=\sin (\pi x) \sin (\pi y) t^{2}$. Table 1 shows the convergence of the method in space for $\tau=\Delta t=0.01$ for fractional orders $\alpha=$ $0.25,0.50,0.75 . N_{x}$ and $N_{y}$ stand for the number of basis in $x$ and $y$ direction. Figure 5.2 demonstrates the logarithmic scale error plot in terms of $H^{1}$-norm for $\alpha=1 / 2$ for some $t$ 's. It is seen that the method preserves the spectral convergence at different time rows $t<1$ and $t>1$. Table 2 reports the convergence in time by considering $N_{x}=N_{y}=N=8$ as $\tau$ decreases at time rows $t=0.1$ and $t=1$. It verifies the $O\left(\tau^{2-\alpha}\right)$ temporal rate of convergence.

Table 1 Convergence in space at $t=1$ for Example 1

\begin{tabular}{ccccccc}
\hline \multicolumn{4}{c}{$\alpha=0.25$} & \multicolumn{2}{c}{$\alpha=0.5$} & \multicolumn{2}{c}{$\alpha=0.75$} \\
\hline$N_{x}=N_{y}$ & $L^{\infty}$ & $H^{1}$ & $L^{\infty}$ & $H^{1}$ & $L^{\infty}$ & $H^{1}$ \\
\hline 2 & $7.53 \mathrm{E}-02$ & $2.81 \mathrm{E}-01$ & $7.52 \mathrm{E}-02$ & $2.81 \mathrm{E}-01$ & $7.49 \mathrm{E}-02$ & $2.81 \mathrm{E}-01$ \\
4 & $1.74 \mathrm{E}-03$ & $8.91 \mathrm{E}-03$ & $1.72 \mathrm{E}-03$ & $8.91 \mathrm{E}-03$ & $1.63 \mathrm{E}-03$ & $8.91 \mathrm{E}-03$ \\
6 & $1.78 \mathrm{E}-05$ & $1.34 \mathrm{E}-04$ & $3.01 \mathrm{E}-05$ & $1.43 \mathrm{E}-04$ & $9.98 \mathrm{E}-05$ & $2.86 \mathrm{E}-04$ \\
8 & $3.67 \mathrm{E}-06$ & $8.75 \mathrm{E}-06$ & $2.25 \mathrm{E}-05$ & $5.15 \mathrm{E}-05$ & $2.79 \mathrm{E}-06$ & $2.54 \mathrm{E}-04$ \\
\hline
\end{tabular}

Example 2 To see the method works for the case in which there is no source term, consider the problem (1.1)-1.3 with the initial condition $u(x, y, 0)=x(x-1) \sin (2 \pi y), \kappa=1$ and no source term [36]. 


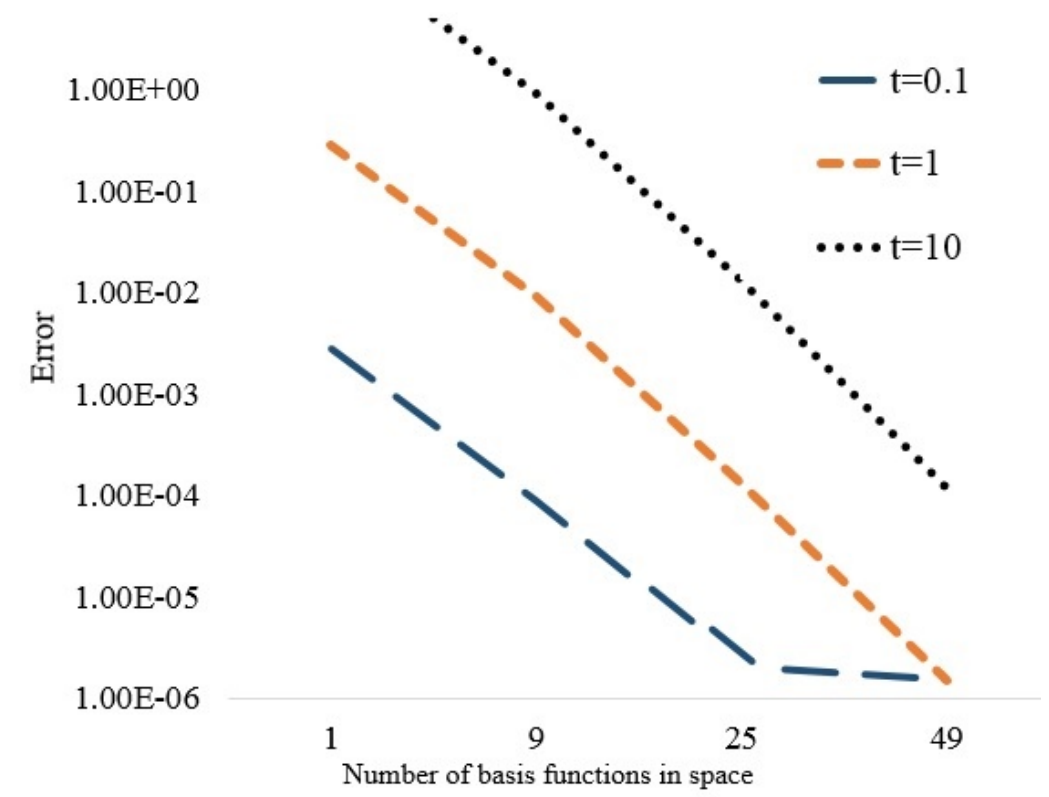

Fig. 5.2 Convergence of the spectral method 3.13 in space with $\tau=0.001$.

Table 2 Error and temporal rate of convergence at $t=1$ for Example 1

\begin{tabular}{|c|c|c|c|c|c|c|c|c|c|c|c|c|}
\hline & \multicolumn{4}{|c|}{$\alpha=0.25$} & \multicolumn{4}{|c|}{$\alpha=0.5$} & \multicolumn{4}{|c|}{$\alpha=0.75$} \\
\hline & \multicolumn{2}{|c|}{$t=0.1$} & \multicolumn{2}{|c|}{$t=1$} & \multicolumn{2}{|c|}{$t=0.1$} & \multicolumn{2}{|c|}{$t=1$} & \multicolumn{2}{|c|}{$t=0.1$} & \multicolumn{2}{|c|}{$t=1$} \\
\hline$M$ & $H^{1}$ & rate & $H^{1}$ & rate & $H^{1}$ & rate & $H^{1}$ & rate & $H^{1}$ & rate & $H^{1}$ & rate \\
\hline 10 & $2.90 \mathrm{E}-04$ & & $4.21 \mathrm{E}-04$ & & $1.16 \mathrm{E}-03$ & & $1.55 \mathrm{E}-03$ & & $3.27 \mathrm{E}-03$ & & $4.46 \mathrm{E}-03$ & \\
\hline 20 & $9.93 \mathrm{E}-05$ & 1.55 & $1.32 \mathrm{E}-04$ & 1.67 & $4.60 \mathrm{E}-04$ & 1.33 & $5.61 \mathrm{E}-04$ & 1.47 & $1.54 \mathrm{E}-03$ & 1.09 & $1.89 \mathrm{E}-03$ & 1.24 \\
\hline 40 & $3.27 \mathrm{E}-05$ & 1.60 & $4.12 \mathrm{E}-05$ & 1.68 & $1.73 \mathrm{E}-04$ & 1.41 & $2.01 \mathrm{E}-04$ & 1.48 & $6.87 \mathrm{E}-04$ & 1.16 & $7.95 \mathrm{E}-04$ & 1.25 \\
\hline 80 & $1.05 \mathrm{E}-05$ & 1.64 & $1.27 \mathrm{E}-05$ & 1.70 & $6.35 \mathrm{E}-05$ & 1.45 & $7.18 \mathrm{E}-05$ & 1.49 & $2.97 \mathrm{E}-04$ & 1.21 & 3.35E-04 & 1.25 \\
\hline 160 & $3.31 \mathrm{E}-06$ & 1.67 & $4.05 \mathrm{E}-06$ & 1.65 & $2.30 \mathrm{E}-05$ & 1.47 & $2.56 \mathrm{E}-05$ & 1.49 & $1.26 \mathrm{E}-04$ & 1.24 & $1.41 \mathrm{E}-04$ & 1.25 \\
\hline
\end{tabular}

The spectral convergence in space is seen from Fig. 5.3 in which the time step length is considered to be $\tau=0.01$. The solution with $N_{x}=N_{y}=10$ is treated as the exact solution. The errors are reported at $t=1$ with $H^{1}$-norm.

The numerical examples present the spectral convergence in space and fixed convergence of $O\left(\tau^{2-\alpha}\right)$ in time confirming the theoretical claims. It should be noted that we have used the eight point Gauss-Legendre quadrature rule to perform the integrals 3.12 in the right hand side of the linear system 3.13 .

\section{Conclusion}

In this paper, some new aspects of the dual Bernstein polynomials have been discussed. A suitable compact combinations of these polynomials has been derived for developing a dual-Petrov-Galerkin variational formulation for the numerical simulation of the two-dimensional subdiffusion equation. It was shown that the method leads to sparse linear systems. The illustrated numerical examples have 


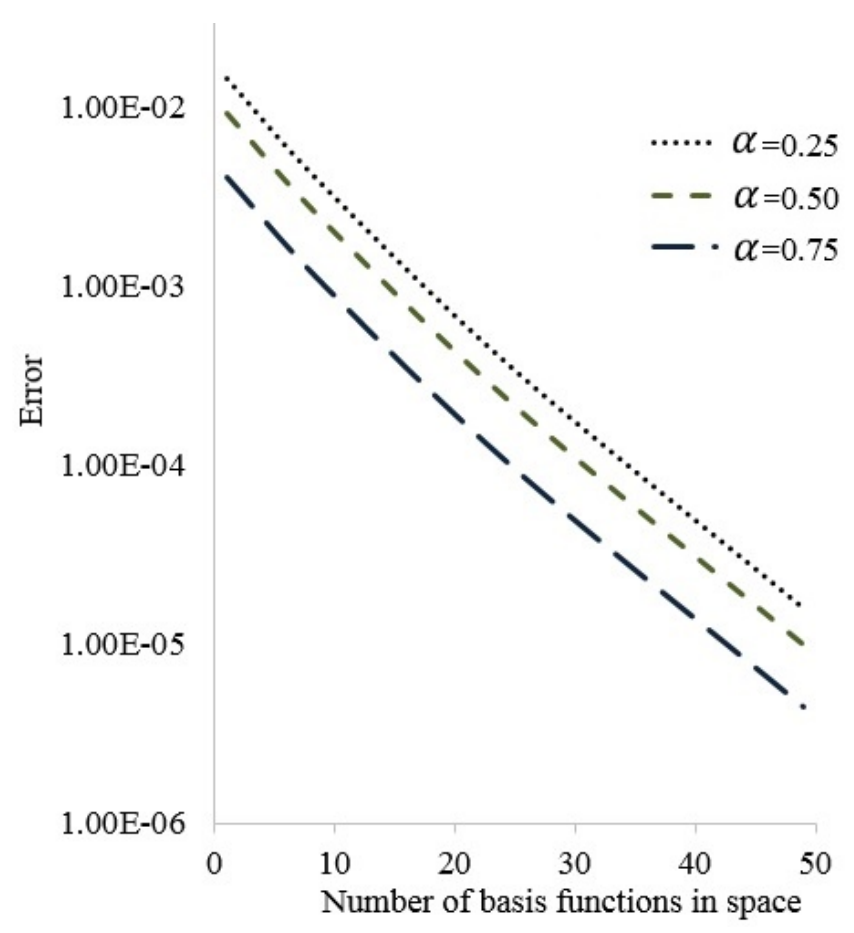

Fig. 5.3 Convergence in space for some fractional orders with $\tau=0.01$.

been provided to show the accuracy of the method. It is important to note that the transformation matrices and the operational matrix for differentiation of dual Bernstein polynomials that have been obtained in this work can be used similarly for developing Bernstein-based dual-Petrov-Galerkin Galerkin methods for other fractional partial differential equations on bounded domains.

\section{References}

1. Behiry, S.H.: Solution of nonlinear Fredholm integro-differential equations using a hybrid of block pulse functions and normalized Bernstein polynomials. J. Comput. Appl. Math 260, 258-265 (2014)

2. Carnicer, J.M., Pena, J.M.: Shape preserving representations and optimality of the Bernstein basis. Adv. Comput. Math 1, 173-196 (1993)

3. Dabiri, A., Butcher, E.A.: Efficient modified Chebyshev differentiation matrices for fractional differential equations. Commun. Nonlinear Sci. Numer. Simul doi: 10.1016/j.cnsns.2017.02.009 (2017)

4. Deng, W.: Finite element method for the space and time fractional Fokker-Planck equation. SIAM J. Numer. Anal 47, 204-226 (2008)

5. Farin, G.E., Hoschek, J., Kim, M.S.: Handbook of computer aided geometric design. Elsevier, Amsterdam (2002)

6. Farouki, R.T., Rajan, V.T.: Algorithms for polynomials in Bernstein form. Comput. Aided Geom. Des 5, 1-26 (1988) 
7. Farouki, R.T.: On the stability of transformations between power and Bernstein polynomial forms. Comput. Aided Geom. Des 8, 29-36 (1991)

8. Farouki, R.T., Goodman, T.N.T.: On the optimal stability of the Bernstein basis. Math. Comput 64, 1553-1566 (1996)

9. Gao, G.H., Sun, Z.Z., Zhang, Y.N.: A finite difference scheme for fractional sub-diffusion equations on an unbounded domain using artificial boundary conditions. J. Comput. Phys 231, 2865-2879 (2012)

10. Gao, G.H., Sun, H.W., Sun, Z.Z.: Stability and convergence of finite difference schemes for a class of time-fractional sub-diffusion equations based on certain superconvergence. J. Comput. Phys 280, 510-528 (2015)

11. Goychuk, I.: Viscoelastic subdiffusion: from anomalous to normal. Phys. Rev. E 80, 046125 (2009)

12. Jani, M., Babolian, E., Javadi, S., Bhatta, D.: Banded operational matrices for Bernstein polynomials and application to the fractional advection-dispersion equation. Numer Algor DOI: 10.1007/s11075-016-0229-1 (2017)

13. Javadi, S., Babolian, E., Taheri, Z.: Solving generalized pantograph equations by shifted orthonormal Bernstein polynomials. J. Comput. Appl. Math 303, 1-14 (2016)

14. Javadi S., Jani M, Babolian E.: A numerical scheme for space-time fractional advection-dispersion equation. Int. J. Nonlinear Analysis Appl 7, 331-343 (2016)

15. Jin, B., Lazarov, R., Zhou, Z.: Two fully discrete schemes for fractional diffusion and diffusionwave equations with nonsmooth data. SIAM J. Sci. Comput 38, A146-A170 (2016)

16. Golub, G.H., Ortega, J.M.: Scientific computing and differential equations: an introduction to numerical methods. Academic Press, San Diego (1992)

17. Goubet, O., Shen, J.: On the dual Petrov-Galerkin formulation of the KDV equation on a finite interval. Adv. Differ. Equ 12, 221-239 (2007)

18. Juttler, B.: The dual basis functions for the Bernstein polynomials. Adv. Comput. Math 8, 345-352 (1998)

19. Lewanowicz, S., Wozny, P.: Bezier representation of the constrained dual Bernstein polynomials. Appl. Math. Comput 218, 4580-4586 (2011)

20. Lin, Y., Chuanju X.: Finite difference/spectral approximations for the time-fractional diffusion equation. J. Comput. Phys 225, 1533-1552 (2007)

21. Maleknejad, K., Basirat, B., Hashemizadeh, E.: A Bernstein operational matrix approach for solving a system of high order linear Volterra-Fredholm integro-differential equations. Math. Comput. Modelling 55, 1363-1372 (2012)

22. Metzler, R., Klafter, J.: The restaurant at the end of the random walk: recent developments in the description of anomalous transport by fractional dynamics. J. Phys. A: Math. Gen 37, R161-R208 (2004)

23. Moghaddam, B.P., Machado, J.A.T.: A stable three-level explicit spline finite difference scheme for a class of nonlinear time variable order fractional partial differential equations. Comput. Math. Appl doi: 10.1016/j.camwa.2016.07.010 (2017)

24. Mustapha, K., Nour, M., Cockburn, B.: Convergence and superconvergence analyses of HDG methods for time fractional diffusion problems. Adv Comput Math 42, 377-393 (2016)

25. Pang, H.K., Sun, H.W.: Fourth order finite difference schemes for time-space fractional subdiffusion equations. Comput. Math. Appl 71, 287-1302 (2016)

26. Ramezani, M., Mojtabaei, M., Mirzaei, D.: DMLPG solution of the fractional advection-diffusion problem. Eng. Anal. Bound. Elem 59, 36-42 (2015)

27. Ren, J., Sun, Z.Z., Zhao, X.: Compact difference scheme for the fractional sub-diffusion equation with Neumann boundary conditions. J. Comput. Phys 232, 456-467 (2013) 
28. Saadatmandi, A.: Bernstein operational matrix of fractional derivatives and its applications. Appl. Math. Model 38, 1365-1372 (2014)

29. Shen, J., Tang, T., Wang, L.L.: Spectral methods: algorithms, analysis and applications. Springer Science \& Business Media, Berlin Heidelberg (2011)

30. Shirzadi, A., Ling, L., Abbasbandy, S.: Meshless simulations of the two-dimensional fractionaltime convection-diffusion-reaction equations. Eng. Anal. Bound. Elem 36, 1522-1527 (2012)

31. Stokes, P.W., Philippa, B., Read, W., White, R.D.: Efficient numerical solution of the time fractional diffusion equation by mapping from its Brownian counterpart. J. Comput. Phys 282, 334-344 (2015)

32. Wang, T., Wang, Y.M.: A compact LOD method and its extrapolation for two-dimensional modified anomalous fractional sub-diffusion equations. Comput. Math. Appl 71, 147-170 (2016)

33. Wozny, P., Lewanowicz, S.: Multi-degree reduction of Bezier curves with constraints, using dual Bernstein basis polynomials. Comput. Aided Geom. Des 26, 566-579 (2009)

34. A new numerical technique for solving the local fractional diffusion equation: Two-dimensional extended differential transform approach. Appl. Math. Comput 274, 143-151 (2016)

35. Yang, J.Y., Zhao, Y.M., Liu, N., Bu, W.P., Xu, T.L., Tang, Y.F.: An implicit MLS Meshless method for 2D time dependent fractional diffusion-wave equation. Appl. Math. Model 39, 1229-1240 (2015)

36. Yang, X., Zhang, H., Xu, Da.: Orthogonal spline collocation method for the two-dimensional fractional sub-diffusion equation. J. Comput. Phys 256, 824-837 (2014)

37. Yuan, J.M., Shen, J., Wu, J.: A dual-Petrov-Galerkin method for the Kawahara-type equations. J Sci Comput 34, 48-63 (2008)

38. Zhang, Y.N., Sun. Z.Z.: Alternating direction implicit schemes for the two-dimensional fractional sub-diffusion equation. J. Comput. Phys 230, 8713-8728 (2011)

39. Zhang, Y.N., Sun, Z.Z., Zhao, X.: Compact alternating direction implicit scheme for the twodimensional fractional diffusion-wave equation. SIAM J. Numer. Anal 50, 1535-1555 (2012)

40. Zhao, Y., Zhang, Y., Shi, D., Liu, F., Turner, I.: Superconvergence analysis of nonconforming finite element method for two-dimensional time fractional diffusion equations. Appl. Math. Lett 59, 38-47 (2016)

41. Zhou, Z., Gong, W.: Finite element approximation of optimal control problems governed by time fractional diffusion equation. Comput. Math. Appl 71, 301-318 (2016) 